\title{
Aislamiento e identificación de Pseudomonas sp. y Aeromonas sp. en aguas de piscinas públicas de Bogotá - Colombia
}

\author{
Isolation and identification of Pseudomonas sp. and Aeromonas sp. In \\ public swimming pool waters of Bogotá - Colombia
}

Estupiñán-Torres Sandra Mónica ${ }^{1}$, Ávila de Navia Sara Lilia' ${ }^{1}$ López Orozco Yeny Lorena ${ }^{2}$, Martínez Méndez Sandra Liliana², Miranda Marín Yeimy Yaritza², Ortegón Puentes Andrea del Pilar ${ }^{2}$

\section{Resumen}

Objetivo. Analizar la calidad del agua de piscinas públicas de Bogotá mediante el recuento de Pseudomonas sp. y Aeromonas sp. Método. Se tomaron 48 muestras de agua de 8 piscinas públicas, durante 3 meses. Se realizó el método de filtración por membrana para el recuento de estos dos géneros bacterianos. Resultados. El 100\% de las piscinas analizadas presentan recuentos de Pseudomonas sp. por encima de lo contemplado en la Resolución 1618 de 2010 (Colombia) y 63\% de las piscinas presentaron recuentos de Aeromonas sp.

Palabras claves: Calidad del agua, piscinas, Pseudomonas, Aeromonas.

\begin{abstract}
Objective. To determine the quality of the water of public swimming pools of Bogota by means of the count of Pseudomonas sp. and Aeromonas sp. Method. It took 48 water samples from eight public pools, for 3 months, was performed by membrane filtration method for the enumeration of these two bacterial genera. Results. The results show that $100 \%$ of the tested pools present counts of Pseudomonas sp. above that provided for in the Resolution 1618 and 63\% of the pools had counts of Aeromonas sp.
\end{abstract}

Keywords: Water quality, swimming pools, Pseudomonas, Aeromonas.

1. Grupo Calidad de aguas. Programa de Bacteriología y Laboratorio Clínico. Universidad Colegio Mayor de Cundinamarca.

2. Egresada Programa de Bacteriología y Laboratorio Clínico. Universidad Colegio Mayor de Cundinamarca. 


\section{Introducción}

Las Pseudomonas y Aeromonas son bacilos Gram negativos, aerobios, oxidasa positivos. Tienen, gracias a su metabolismo, una cierta facilidad de adaptación que les permite adecuar el hábitat donde se encuentren para utilizar diferentes fuentes como el carbono o el nitrógeno para su nutrición (1). Poseen una cápsula de exopolisacáridos que facilita la adhesión celular, la formación de biopelículas y las protege de la fagocitosis o los iones libres formados en la potabilización del agua, aumentando así su patogenicidad $(1,2)$. Debido a esta adaptación al medio ambiente las Pseudomonas y Aeromonas se han convertido en un problema para la salud pública ya que estos microorganismos son capaces de colonizar un amplio rango de nichos dentro de los cuales se encuentra el agua de piscina que, a pesar de ser tratada mediante procesos de desinfección, presenta estas bacterias que causan patologías de consideración en los usuarios (3).

En este sentido, el objetivo de aislar e identificar Pseudomonas (incluidas en la Resolución 1618 de 2010) y Aeromonas (no incluidas en la Resolución), en agua de piscinas públicas de la ciudad de Bogotá busca plantear la necesidad de establecer indicadores bacterianos no habituales que puedan definir mejor la calidad del agua para uso recreacional, con el fin de demostrar que los indicadores microbiológicos utilizados tradicionalmente para evaluar los riesgos asociados al uso de las aguas de piscinas no siempre suministran una información suficiente de los riesgos reales del usuario.

\section{Materiales y métodos}

Se estudiaron ocho piscinas públicas de la ciudad de Bogotá, identificadas alfabéticamente de la A-H. En cada una se tomaron dos muestras, una en las horas de la mañana y otra en horas de la tarde. Se realizó un muestreo por mes (abril, mayo y junio) del año 2009. Para la recolección de las muestras se utilizaron frascos Schott de $250 \mathrm{~mL}$ con Tiosulfato de Sodio al 10\%. La muestra se tomó a $50 \mathrm{~cm}$ de la superficie introduciendo el frasco en posición invertida y fueron transportadas a $4^{\circ} \mathrm{C}$.

Para el procesamiento de las muestras se utilizó la técnica de filtración por membrana según los procedimientos del Standard Methods (4) y de USEPA (5). Como control se utilizaron cepas de Pseudomonas aeruginosa ATCC 9027 y de Aeromonas hydrophila ATCC 1020. La identificación de algunas de las bacterias aisladas se realizó mediante BBL Crystal E/NF.

\section{Resultados}

En el primer muestreo realizado en el mes de abril, el $88 \%$ y el $100 \%$ de las muestras presentaron recuentos de Pseudomonas spp. en horas de la mañana y la tarde respectivamente. Para el mes de mayo se obtuvieron recuentos de Pseudomonas sp. en el $100 \%$ de las piscinas tanto en horas de la mañana como en la tarde. En el tercer muestreo, realizado en el mes de junio, se presentó Pseudomonas en el $100 \%$ de las piscinas tanto en horas de la mañana como en horas de la tarde. El recuento en siete de las piscinas a lo largo de los tres meses varía, mientras que en la piscina $G$ los resultados fueron los mismos en los tres meses tanto en la mañana como en la tarde, Tabla 1. Estos resultados indican que ninguna de las piscinas cumple con lo establecido con la Resolución 1618/10, según la cual el recuento de este microorganismo debe ser 0 UF$\mathrm{C} / 100 \mathrm{~mL}$.

Tabla 1. Recuentos de Pseudomonas spp. en UFC/100mL.

\begin{tabular}{|l|r|r|r|r|r|r|}
\hline \multirow{2}{*}{ Piscina } & \multicolumn{2}{|c|}{ ABRIL } & \multicolumn{2}{|c|}{ MAYO } & \multicolumn{2}{|r|}{ JUNIO } \\
\cline { 2 - 7 } & Mañana & Tarde & Mañana & Tarde & Mañana & Tarde \\
\hline A & 100 & 100 & 5 & 35 & 10 & 30 \\
\hline B & 23 & 15 & 100 & 100 & 100 & 1 \\
\hline C & 100 & 100 & 5 & 10 & 52 & 23 \\
\hline D & 100 & 100 & 62 & 30 & 100 & 100 \\
\hline E & 100 & 100 & 7 & 5 & 100 & 100 \\
\hline F & 0 & 18 & 45 & 100 & 58 & 33 \\
\hline G & 100 & 100 & 100 & 100 & 100 & 100 \\
\hline H & 10 & 100 & 3 & 3 & 34 & 6 \\
\hline
\end{tabular}


Como se observa en la Figura 1, durante los tres meses del estudio el recuento promedio más alto fue el de la piscina G con $100 \mathrm{UFC} / 100 \mathrm{ml}$ y el más bajo el de la piscina $\mathrm{H}$ con $26 \mathrm{UFC} / 100 \mathrm{~mL}$.

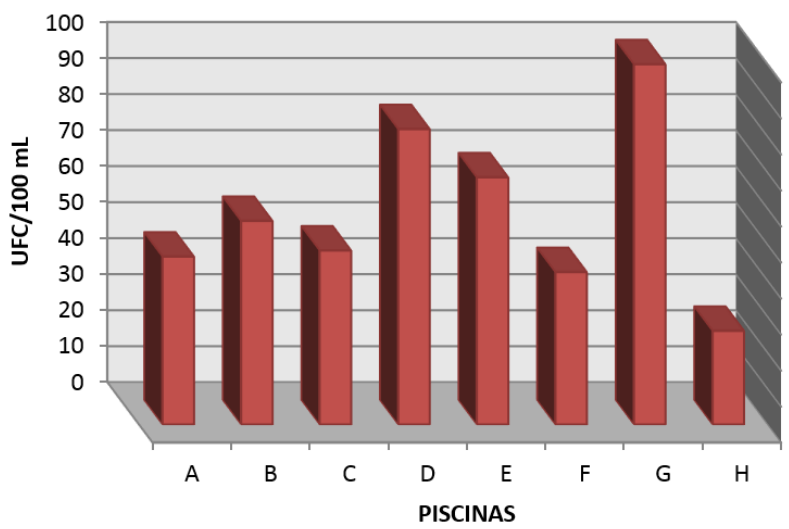

Figura 1. Recuento promedio en UFC/100mL de Pseudomonas spp.

En la Figura 2 se observa que el recuento promedio de Pseudomonas spp. es más bajo en la mańana durante los meses de abril y mayo, mientras que en el mes de junio es más alto en la mañana.

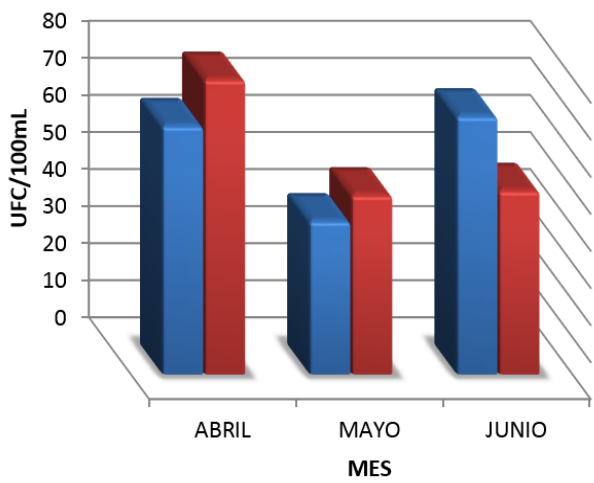

$\square$ MAÑANA - $T$ TARDE

Figura 2. Recuento promedio de Pseudomonas spp. en mañana y tarde.z

En relación a las Aeromonas sp., el 13\% de las piscinas presentó recuentos en los muestreos de la mańana y la tarde. En el mes de abril únicamente se obtuvo recuento de la piscina $\mathrm{H}$ tanto en la muestra de la mańana como la de la tarde; las piscinas $\mathrm{A}, \mathrm{B}, \mathrm{C}, \mathrm{D}, \mathrm{E}, \mathrm{F}$ no presentaron UF$\mathrm{C} / 100 \mathrm{~mL}$ en ninguno de los muestreos realizados en el día, Tabla 2. En el mes de mayo, la piscina $G$ presentó un recuento en horas de la mańana mayor que el de las horas de la tarde para Aeromonas sp. mientras que las piscinas $\mathrm{F}$ y $\mathrm{H}$ únicamente presentaron recuentos en horas de la tarde. Sin embargo, los recuentos obtenidos son bajos y en las piscinas A, B, C, D y E fue de $0 \mathrm{UFC} / 100 \mathrm{~mL}$ para éste microorganismo (Tabla 2). El 13\% de las piscinas presentaron recuentos de Aeromonas sp. en horas de la mańana y el 38\% en la tarde (Tabla 2). Para el mes de junio de 2009 las piscinas $C$ y D presentaron recuentos de Aeromonas sp. en horas de la mańana únicamente, la piscina $\mathrm{B}$ presentó recuento para este microorganismo únicamente en horas de la tarde, mientras que las piscinas $\mathrm{F}$ y $\mathrm{H}$ presentaron recuentos tanto en horas de la mańana como en horas de la tarde, Tabla 2.

Tabla 2. Recuentos de Aeromonas sp. en UFC/100mL.

\begin{tabular}{|l|r|r|r|r|r|r|}
\multirow{2}{*}{ Piscina } & \multicolumn{2}{|r|}{ ABRIL } & \multicolumn{2}{|r|}{ MAYO } & \multicolumn{2}{|r|}{ JUNIO } \\
\cline { 2 - 7 } & Mañana & Tarde & Mañana & Tarde & Mañana & Tarde \\
\hline A & 0 & 0 & 0 & 0 & 0 & 0 \\
\hline B & 0 & 0 & 0 & 0 & 0 & 1 \\
\hline C & 0 & 0 & 0 & 0 & 3 & 0 \\
\hline D & 0 & 0 & 0 & 0 & 1 & 0 \\
\hline E & 0 & 0 & 0 & 0 & 0 & 0 \\
\hline F & 0 & 0 & 0 & 1 & 5 & 2 \\
\hline G & 0 & 0 & 8 & 1 & 0 & 0 \\
\hline H & 15 & 10 & 0 & 7 & 3 & 6 \\
\hline
\end{tabular}

Durante los tres meses del estudio, el recuento promedio más alto fue la piscina $\mathrm{H}$ con $6.83 \mathrm{UF}$ $\mathrm{C} / 100 \mathrm{~mL}$ y el más bajo el de las piscinas A y E con $0 \mathrm{UFC} / 100 \mathrm{~mL}$, Figura 3.

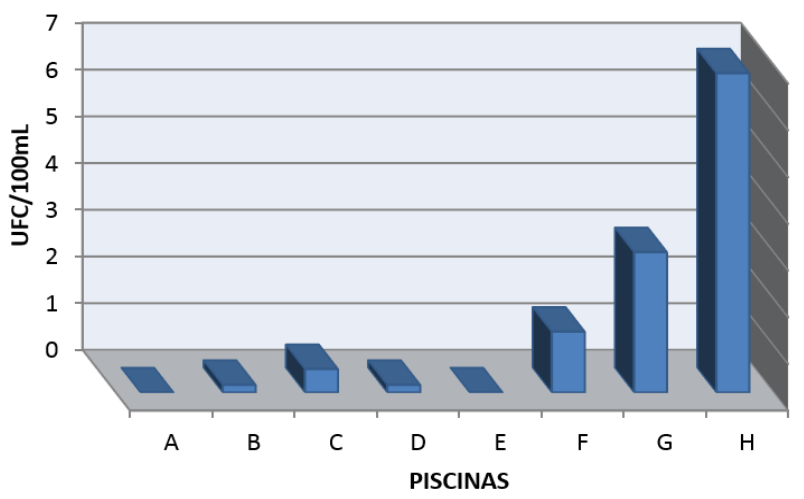

Figura 3. Recuento promedio en UFC/100mL de Aeromonas sp. 
En la Figura 4, se observa que el recuento promedio de Aeromonas sp. es más bajo en la mañana en mayo, mientras que en abril y junio es más alto en la mañana.

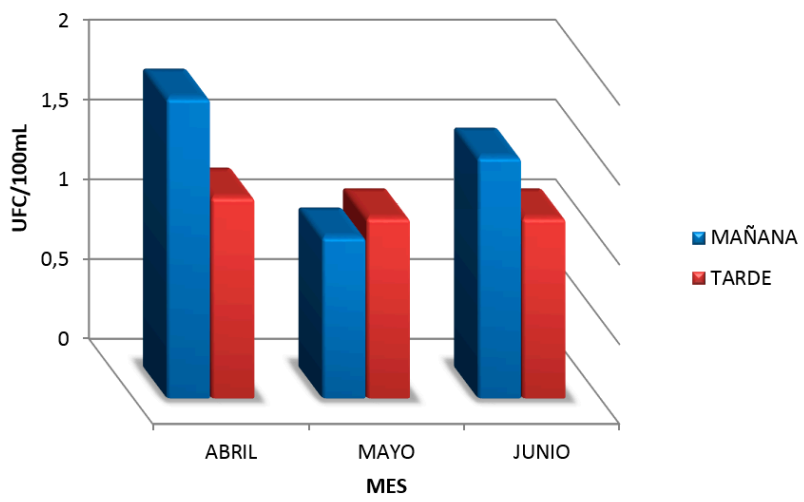

Figura 4. Recuento promedio de Aeromonas sp. en mañana y tarde.

La identificación se realizó en colonias aisladas escogidas al azar por el método BBL Crystal E/ NF. Los microorganismos identificados fueron Pseudomonas aeruginosa, presente en todas las piscinas y Stenotrophomonas maltophilia, aislada de las piscinas $\mathrm{B}$ y $\mathrm{G}$.

En cuanto a el género de Aeromonas sp. fue identificado en el 63\% de las piscinas, con bajos recuentos, pero no fue posible la identificación de la especie por el difícil aislamiento que esta presenta.

Las pruebas microbiológicas que se realizaron por la administración de las piscinas fueron: en el mes de abril coliformes fecales, en el mes de mayo coliformes fecales y totales y en junio coliformes fecales. Según los reportes suministrados todas las piscinas cumplen con lo establecido por la legislación (0 UFC/100mL) (6).

\section{Discusión}

Pseudomonas aeruginosa es capaz de sobrevivir y multiplicarse en aguas tratadas (1), debido a que necesita pequeñas cantidades de nutrientes y posee una capa polisacárida, que constituye una barrera fisicoquímica que protege a la bacteria de los iones de cloro libre residual (7), por eso su presencia en aguas de piscina cada vez es más frecuente (2).

La presencia de microorganismos causantes de patologías en el agua de uso recreativo ha sido objeto de estudio en varios países como Venezuela donde se sugiere la inclusión de bacterias no habituales como el grupo Coliformes, especies de Pseudomonas, Streptococcus, Staphylococcus y Legionella. En España, las normas que rigen la calidad de aguas de piscinas incluyen a la Pseudomonas aeruginosa como parámetro rutinario, ya que su presencia es en cierto modo un indicador de la eficacia de la cloración, debido a que su resistencia al cloro es superior a la de los otros microorganismos presentes en el agua (8). Es probable que, aunque los niveles de cloro residual libre estén dentro de los parámetros exigidos por la Legislación colombiana, éstos no sean lo suficientes para eliminar totalmente microorganismos patógenos como Pseudomonas aeruginosa.

Los resultados microbiológicos aportados por la administración de las piscinas reportan que los coliformes totales y $E$. coli presentan recuentos de $0 \mathrm{UFC} / 100 \mathrm{~mL}$, lo cual puede indicar que el proceso de desinfección es adecuado para la eliminación de estos microorganismos, pero no para Pseudomonas aeruginosa, o que la presencia de esta última está inhibiendo el crecimiento de los otros indicadores, pues se ha reportado que catabolitos de Pseudomonas aeruginosa, como la piocianina, tienen un efecto bactericida sobre coliformes principalmente sobre E. coli, lo cual produciría su disminución, conduciendo a resultados erróneos en el control de calidad del agua (9).

Los indicadores que normalmente se analizan en el agua de piscinas únicamente evaluarían el proceso de desinfección, mientras que estudios realizados reportan que la determinación de Pseudomonas aeruginosa y Aeromonas sp. podrían ser un indicador de riesgo para la salud del usuario (2, 3, $10)$, ya que se conoce que éstos pueden causar di- 
ferentes enfermedades en los usuarios de las piscinas como otitis externa, conjuntivitis, dermatitis, septicemia, bacteremia, infección de heridas, o foliculitis $(11-14)$. Estudios han reportado cepas de Pseudomonas aeruginosa aisladas de piscina con multiresistencia a antimicrobianos $(15-21)$.

\section{Agradecimientos}

\section{A Yeny Lorena López Orozco, Sandra Liliana Martínez Méndez, Yeimy Yaritza Miranda Marín, Andrea del Pilar Ortegón Puentes, quienes en ca- lidad de estudiantes del Grupo Calidad de Aguas participaron en este proyecto.}

\section{Referencias}

1. Willey J, Sherwood L, Woolverton CJ. 2013. Prescott's Microbiology. ISBN: 978-0073402406

2. Salazar I, y otros. 2004. Indicadores bacterianos no habituales de la calidad de agua en piscinas. Venezuela. Congreso Interamericano de Ingeniería Sanitaria y Ambiental, San Juan, 22-27. Disponible en: http://www.ingenieroambiental. com/4014/salazar.pdf

3. Guida M, Gallé, F Mattei M, Anastasi, D Liguori G.2009. Microbiological quality of the water of recreational and rehabilitation pools: A 2-year survey in Naples, Italy. Public Health 123, 448-451.

4. Franson, M. 1992. Standard methods: for the examination of water and wastewater. Washington. American Public Health.

5. USEPA. 2001. Method 1605: Aeromonas in Finished Water by Membrane filtration using Ampicillin-Dextrin Agar with Vancomycin (ADA-V). Washington, DC.

6. Colombia. Ministerio de la Protección Social. Resolución 1618 de 2010, Bogotá, D.C. 2010; 1-16

7. Van den Akker R, Pomati F, Roser D. 2012. A risk assessment of Pseudomonas aeruginosa in swimming pools: A review. J. Water. Health. 10: 181-196.

8. Real Decreto 742/2013, de 27 de septiembre, por el que se establecen los criterios técnico-sanitarios de las piscinas. Ministerio De Sanidad, Servicios Sociales E Igualdad, España.

9. Vaconcelos U, Gomes M \& Torres G. 2010. Pseudomonas aeruginosa associated with negative interactions on coliform bacteria growth. Canadian Journal of pure $\&$ applied Sciences, 4: 1133-1139

10. Saegeman V, Van Meensel B.2016. Aeromonas Associated with Swimming Pool Folliculitis. Pediatr Infect Dis J. Jan;35(1):118-9.
11. Koneman E, Allen S, Janda W. 2008. Diagnóstico Microbiológico. Argentina. Médica Panamericana.

12. Castro E, Aguilera AM. 2002. El género Aeromonas ¿¿Un patógeno importante en México? Enfermedades Infecciosas y Microbiología. 22: 206 .

13. González M, et al. 2004. Aeromonas sp.: patógenos emergentes a considerar en aguas. Medio Ambiente y Desarrollo; Revista electrónica de la Agencia de Medio Ambiente. Año 4, No. 6, ISSN:1683-8904

14. Estupiñan SM, Avila SL. Calidad fisico química y microbiológica del agua del municipio de Bojaca, Cundinamarca. NOVA. 2010; 8(14): 206-212.

15. Lutz JK, Lee J. 2011. Prevalence and Antimicrobial-Resistance of Pseudomonas aeruginosa in Swimming Pools and Hot Tubs. Int. J. Environ. Res. Public Health. 8: 554-564.

16. Papadopouloua C, et al. 2008. Microbiological quality of indoor and outdoor swimming pools in Greece: Investigation of the antibiotic resistance of the bacterial isolates. International Journal of Hygiene and Environmental Health. 211 (3-4): 385-397.

17. Estupiñan SM, Avila SL. Calidad sanitaria del agua del parque natural Chicaque. NOVA. 2013; 11(20): 45 - 51.

18. De Navia SLÁ, Torres SME. Calidad sanitaria del agua del Parque Natural Chicaque. Nova. 2013;11(20).

19. Ramírez, L. C. C., et al. Determinación de la presencia de bacterias patógenas para el humano en aguas de riego en la cuenca alta de la sabana de Bogotá; DC Colombia. Nova. 2014; 12(22)

20. Corrales, L. C., et al. Bacterias anaerobias: procesos que realizan y contribuyen a la sostenibilidad de la vida en el planeta. 2015; Nova 13(24): 55-82.

21. Rodríguez, O. E., Andrade, W. A., Díaz, F. E., \& Moncada, B. Actividad antimicrobiana de líquenes de la cuenca alta del rio Bogotá. 2015; Nova, 13(23). 